\title{
SYSTEMATIC METHODS FOR KNOWLEDGE ACQUISITION AND EXPERT SYSTEM DEVELOPMENT
}

\author{
Brenda L. Belkin and Rober F. Stengel ${ }^{\text {*t* }}$ \\ Princeron University \\ Deparment of Mecharical \& Aerospace Engineering \\ Princeton, NJ, 08544
}

\section{ABSTRACT}

Nine cooperating rule-based systems, collectively called AUTOCREW, were designed to automate functions and decisions associated with a combat aircraft's subsystems. The organization of tasks within each system is described; performance metrics were developed to evaluate the workload of each rule base, and to assess the cooperation berween the rule-bases.

Each AUTOCREW subsystem is composed of several exper systems that perform specific tasks. AUTOCREW's NAVIGATOR was analyzed in detail to understand the difficulties involved in designing the system and to identify tools and methodologies that ease development. The NAVIGATOR determines optimal navigation strategies from a set of available sensors. A Navigation Sensor Management (NSM) exper system was systernatically designed from Kalman Filter covariance data; four ground-based, a satellite-based, and two on-board INS-aiding sensors were modelled and simulated to aid an INS. The NSM Expert was developed using the Aralysis of Variance (ANOVA) and the $\mathbb{D}_{3}$ algorithm. Navigation strategy selection is based on an RSS position error decision metric, which is computed from the covariance data. Results show that the NSM Exper predicts position error correctly between $45 \%$ and $100 \%$ of the time for a specified navaid configuration and aircraft trajectory. The NSM Expert adapts to new siruations, and provides reasonable estimates of hybrid perfomance. The systematic nature of the ANOVA/ID3 meshod makes it broadly applicable to expert system design when experimental or simulation data is available.

\section{INTRODUCTION}

The computational and symbolic processing requirements for pilotaiding systems pose many problems, e.g., multi-task scheduling and intersystem cooperation [1,2]. Expert systems, which are computer programs usually developed in a symbolic processing language such as LISP or PROLOG, have emerged to solve difficult domain-specific problems [3]. The designer generally extracts heuristics and specific knowledge from domain experts. This information is used to formulate a knowledge base consisting of parameters and rules. An inference engine uses rules to conduct a search and to set parameters, thereby inferring lanowledge about the problem domain.

Problem identificarion in the flight environment and expert system development has led to the design and implementation of exper systems in the areas of navigation, emergency procedures, and air traffic control [4-6]. The introduction of multiple system concepts such as global blackbaard architectures for information exchange between knowledge bases has been successful in aerospace implementations [2,7]. However, methods in multiple knowledgebase development, systems integracion, and ensemble protoryping need further research if complex systems are to be developed for flight domain operations or other problem areas.

A logical task classification scheme is a key factor in the successful development of multiple rule-based systems. In this paper a logical

- Fomerty. Graduate Student Princenon Universiry. Currently, Member of Technical Suar, AT T Tell Laborascries. 480 Red Hill Rosd. Middlewow. NI. 07748

- Professar of Mechrical A Aerospace Enginoering

Presented at the 29th DEEE Conference on Decision

and Control, Honolulu, H. December 5-7, 1990. structure for providing assistance to the pilot of a military aircraft was pattemed on the functions of a World War II bomber crew [8]. Based on this model, an ensemble of nine cooperating rule-based systems called AUTOCREW $[9,10]$ was developed. Each sy'srem rigurauvely emulates a crew member's task responsibilities.

To illustrate some of the issues involved in the design of an AUTOCREW subsystem, the problem of navigation sensor selection was srudied in detail. With a large number of available navaid sensors, choosing an optimal or near-optimal sensor set becomes a large combinatorial problem. Convergence towards an optimal sensor configuration requires an exhausive compurer search udilizing simulation results as the basis for selection. In contrast, a small number of available navaids reduces the decision space consicerably. Hence, a dilemma occurs; increasing sensor capabiliry (and thus reliability and performance) increases decision-making complexiry.

A Navigation Sensor Management (NSM) Expert System that controls the selection of multi-sensor configurations was developed for AUTOCREW. Two on-board navigation aids (Dopoler radar and Air data sensor) and five radio navigation systems (Global Positioning System, LORAN, TACAN, DME, and VOR) were modelled and covariance results obtained using a U-D implementation of the Kalman Filter equations [9]. Up to three ground starions and four satellites were simulated for typical high-performance, commercial jet transport. and general aviation aircraft trajectories. Mixed sensor simulations also were performed. These results provided the combinatorial, mulkifacior source required for knowledge-base development.

The Analysis of Variance (ANOVA) stadistical technique [13] was applied to the covariance results to identify the factors that cause variation in navigation performance. Once the important fastors were identified, the relationships berween them were determined. The $\mathbb{D} 3$ algorithon [14], an inductive inference technique based on the probabilistic occurrence of events, was used to find these atribute relationships. Details of the development of the NSM Expert are described in Ref. 9 , and the main results are shown in this paper.

\section{AUTOCREW DESCRIPTION}

The nine AUTOCREW components $[9,10]$ are COPLOT (Ilight control, aircraft performance, terrain following), ENGINEER (system diagnosis, reconfiguracion, emergency procedures). NA VIGATOR (navigation sensor managewent, dynamic route planning), COMMMUNICATOR (radio/data operations), OBSER VER (lookout and alam, identification friend or foe) ATTACKER (offensivi weapon control, iarget acquisition/prioritization). DEFENDER (dếensive weapon control), and SPOOFER (electronic measures/countermeasures). The ninth rule-based system, the EXECUTIVE, coordinates missionspecific tasks and has knowledge of the mission plan. In the AUTOCREW scheme, the human aircraft Pilot acts in the capaciry of mission coordinator. The Pilot has full control of the aircraft and its on-board systems; AUTOCREW can provide automatic assistance when requested. In total, the AUTOCREW system performs 511 tasks, is comprised of 406 rules, and uses 89 shared parameters [9].

\section{Knomledge-Base Development of AUTOCREW Comp- onents}

Five main task groups are performed by each AUTOCREW component: 1) tasks execured during an attack on the aircraft, (2) tasks executed during aircrafi subsystem emergency or polencial threat 


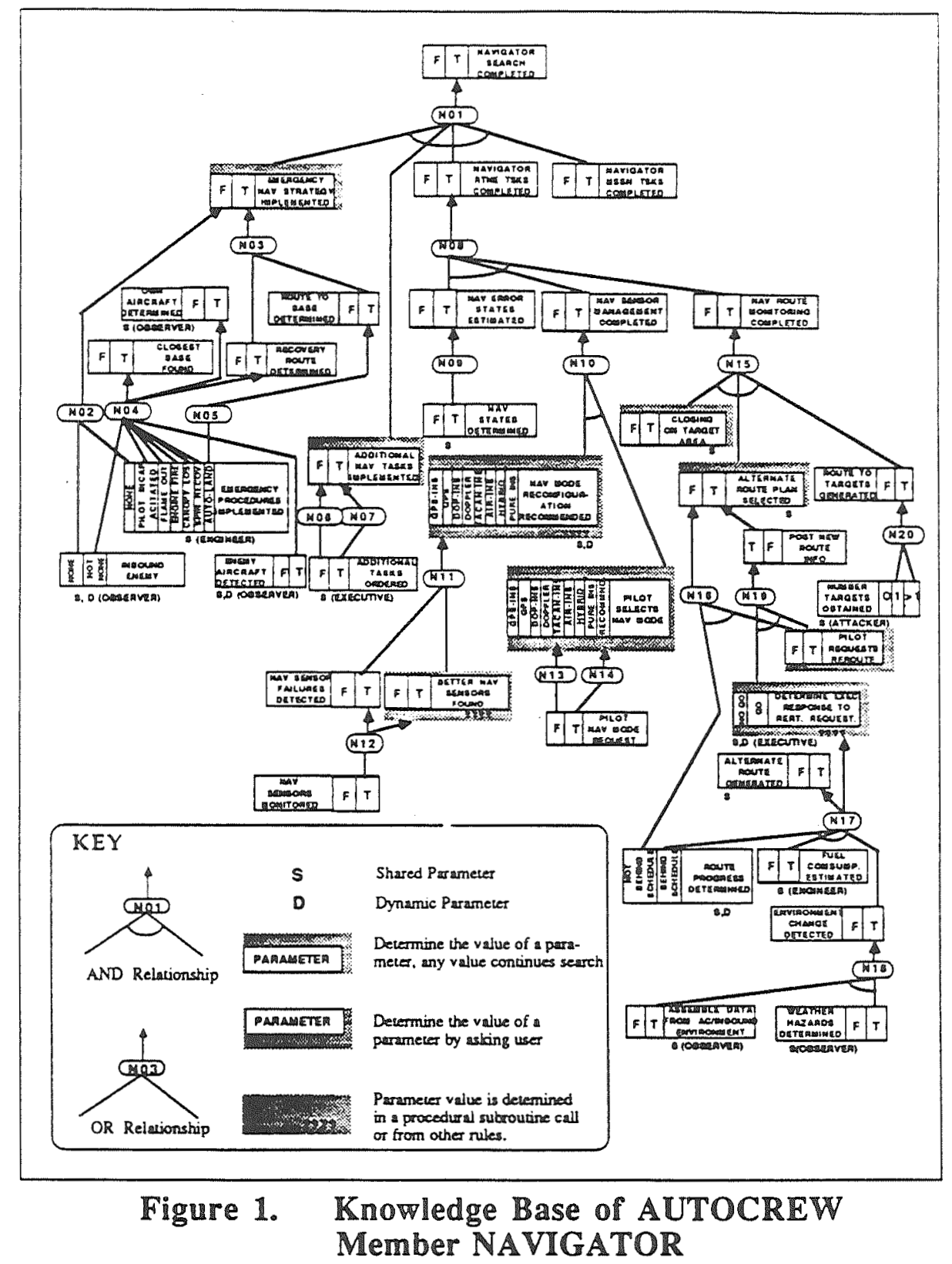

siruations, (3) additional tasks ordered by the EXECUTTVE, (4) tasks executed on a routine basis, and (5) mission-specific tasks that (for the most part) are execused once and that require a high degree of cooperation among the various systems. Mission-specific tasks are divided into groups appropriate to each mission phase [10].

Figure 1 shows the graphical representation of AUTOCREW's NAVIGATOR lnowledge base (see Refs. 9 and 10 for details of the AUTOCREW knowledge bases). The NAVIGATOR's tasks include navigation sensor management, navigation state and error estimation, and dynamic route planning. As seen in the figure, detection of an inbound enerry ariggers the NAVIGATOR to perform defensive tasks. Rule NOA shows that the NAVIGATOR finds the nearest friendly airfield able to serve the aircraft from its stored airbase information.

The NAVIGATOR also uses the OBSERVER's information on friendly aircraft in the area and plans a recovery route. It is extremely imporang that the aircraft's location is known accurately in order to avoid such danger zones as Surface-to-Air Missile (SAM) beits. Tactical aircraft carry inertial navigation systems (INS) for highfrequency navigation information. A Kalman Filter can be used to integrate external navaids with an INS to estimate navigation errors. The insegrased or hybrid INS gives accurate high-frequency navigation information with bounded errors in the state estimates (Rule N09). The error magnitudes depend on the accuracy of the navaid selected. The NAVIGATOR monitars ground-based and satellite radio navaids 

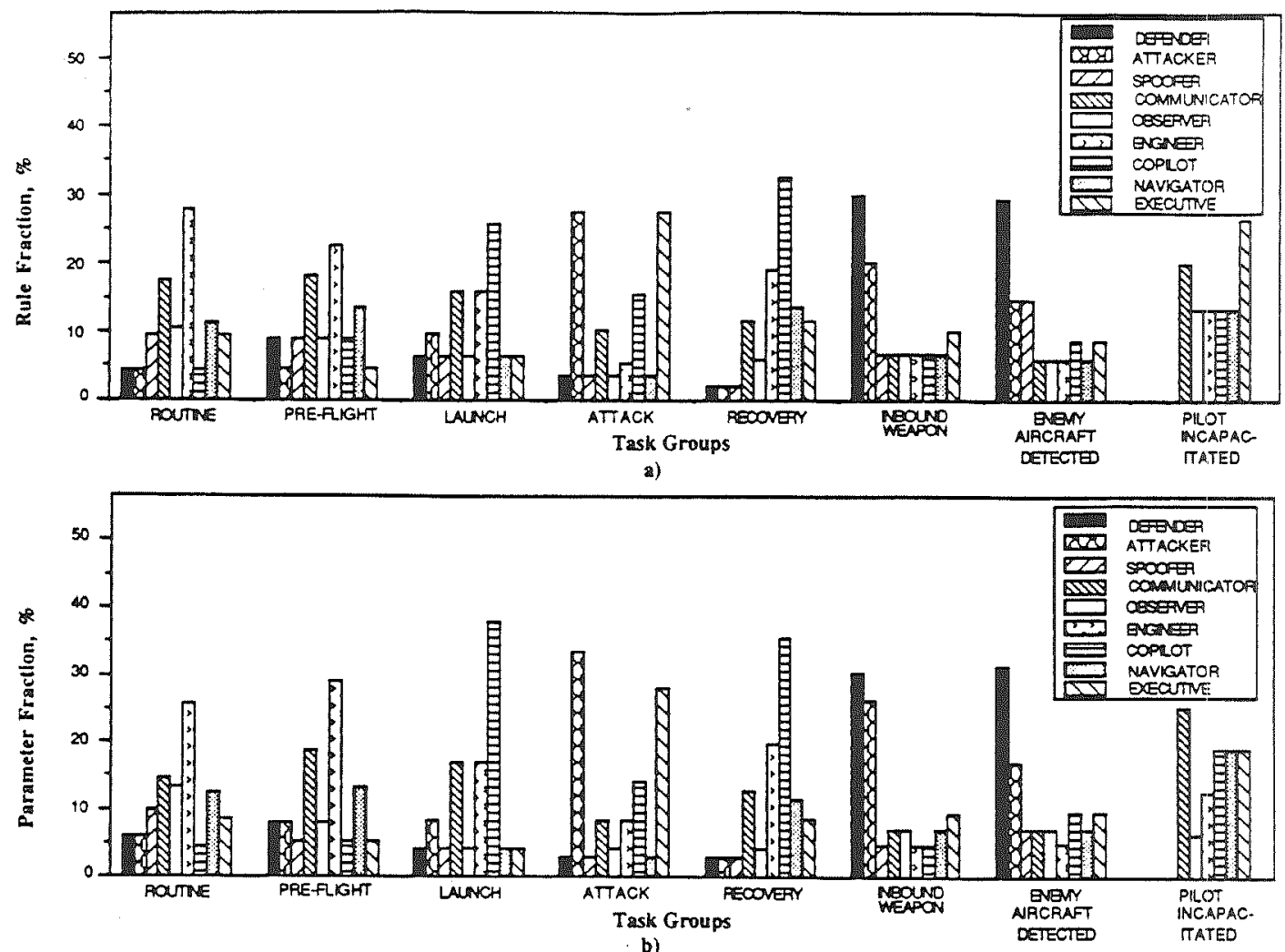

Figure 2. AUTOCREW Workload - Mission-Specific Tasks and Emergency Scenarios

in the area to determine if they are sufficiently operational for integration purposes (Rule 11). From this list of navaids, the NAVIGATOR recommends the best combination meeting the computational resource constraints. The Pilot can change the NAVIGATOR's selection if desired (Rule N14).

The NAVIGATOR also is responsible for routinely monitoring flight plan progress. The NAVIGATOR first determines if the aircraft is closing on the target area. This information initiates additional tasks in the remaining AUTOCREW knowledge bases. The NAVIGATOR keeps a record of aircraft positions, restricted zones, and areas of weather disturbances as reported by the OBSERVER (Rule N18). The NAVIGATOR generates an alternate flight path for the following conditions: flight path conflicts, flight plan behind schedule, and fuel considerations. The EXECUTIVE is consulted when the NAVIGATOR recommends an alternate route. The EXECUTTVE considers the effect of the route change on the overall mission plan and aircraft's safety, and it makes a GO/NO GO recommendation (Rule N17). The Pilot can accept or reject the EXECUTIVE's recommendation in Rule N19. If the new route is selected, the NAVIGATOR posts the route plan details on the display. As noted above, the COPLOT changes its steering commands in response to the route change. When the ATTACKER acquires enemy targets and the plan is to engage, the NA VIGATOR generates roures to each target, as seen in Rule N20.

\section{AUTOCREW Simulation Results}

Simulation and comparative workload results for two mission scenarios are given in this section. The scenarios are inbound SAM attack on the aircraft and Pilot incapacitation. Results are presented for five mission phases (routine, pre-flight, launch, attack, and recovery) and three emergency conditions (inbound weapon, enemy aircraft detected, and pilot incapacitated).

AUTOCREW workloads for three emergency conditions were obtained and compared with the routine workload, referred to as "cruise mode" (Table I). For the three scenarios the workload progressively increases as the situation gets worse. There is a dramatic increase in workload between cruise and attack modes with an inbound SAM, and there is an additional increase betwein the inbound SAM scenario (cruise), and inbound SAM with the: Pilot incapacitated (cruise). The workload nearly doubles for an intound SAM when the aircraft is already engaging another aircraft (attack mode).

\section{Table I Scenario Workload-lncrease Comparisons}

\section{Scenario}

Workload Increase, \% Tasks Decision-Making

\section{Cruise Mode}

Inbound SAM/Cruise Mode

Inbound SAM \& Pilot Incapacirared/Cruise Mode

Inbound SAMAttack Mode

\begin{tabular}{ll}
\hline 26.3 & 28.1 \\
39.5 & 38.6 \\
77.2 & 75.2
\end{tabular}

A comparison of the workload distribution among the AUTOCREW members for each of the given mission phases is shown in Fig. 2. The Rule Fraction is the ratio of number of rales fired during the mission phase for the specified AUTOCREW member to the total number of mission phase rules in all AUTOCREW knowledge bases [9]. The Parameter Fraction is the ratio of number of tasks performed during the mission phase for the specified AUTOCREW component to the total number of mission phase tasks in all AUTOCREW knowledge bases. These metrics were used to characterize the knowledge base 
workloads. The ENGIMEER, COMMUNICATOR, OBSERVER, NAVIGATOR and EXECUTIVE have the largest amount of routine lasks to perform each search cycle. During the pre-flight phase, the ENGINEER, COMMUNICATOR, and NAVIGATOR are the busies components, as each prepares the aircraft for takeoff. About $40 \%$ of all the launch phase tasks are performed by the COPILOT, as shown in Fig. 2, while the next largest task load is executed by the COMMUMICATOR and ENGINEER (each having 17\% of the total launch workload). Most of the artack phase work is done by the ATTACKER (33\%), whereas the recovery phase is dominated by the COPIOT's (35\%) activities. Most of the workload during an inbound weapon atrack is performed by the DEFENDER (about $30 \%$ in Fig. 2). The ATTACKER's workload also increases at this time, as it monitors the DEFENDER's firepower capability.

There is evidence of more SPOOFER decision-making when an enemy aircraft is detected than when the OBSERVER detects an inbound weapon. This is due to its Electronic Counter Measures (ECM) strategy consultation with the EXECUTIVE. The COPIOT workload also increases during these two emergency conditions; in both scenarios, the COPLOT selects an appropriate evasive maneuver. These selections are based on the aircraft capability information provided by the ENGINEER. The EXECUTIVE's workload also increases at this time to assist the Pilot in making decisions. When the Pilor is incapacitated, the EXECUTTVE becomes the primary decision-maker, as shown in Fig. 2. The ENGINEER's major task during this scenario is to monitor the Pilot's condition, resulting in a $10 \%$ workload increase.

\section{DEVELOPMENT OF A NAVIGATION SENSOR} MANAGEMENT EXPERT SYSTEM

The remainder of this paper focuses on the issues involved in designing the navigation sensor selection expert in NAVIGATOR. This example demonstrates how simulation data and statistical analysis can be used to systematically design an expert system.

To determine the performance of the various hybrid navaid combinations, the following U-D Kalman filter simulations were performed:

1. Single-type hybrids: GPS, LORAN, TACAN, DME, VOR, Doppler Radar, or Air Data Sensor aiding an INS

2. Number of stations used in a single-rype hybrid

3. Muli-type hyorids: Combinations of different navaid types aiding an INS

4. Aircraft trajestories simulated: High-performanoe, commercial, general aviation

The root sum of squares (RSS) of the north and east component errors was selected for the hybrid system performance metric. The primary function of this expert system is to select the external navaid sensors that provide the smallest possible RSS position error from a large ser of available sensors. The Analysis of Variance (ANOVA) technique [13] is used to idenuify the factors that make statisrically significant contributions to the decision metric. Then, the $\mathrm{ID} 3$ algorithrn deternoines the relacionships berween these factors $[13,14]$.

\section{Identifying Important Factors Using ANOVA}

The RSS position error histories from over 200 covariance simulations were obcained, and the data were used in an ANOVA four-factor navaid experiment. The goal of the experiment was to identify which of the factors (navaid rype, number of ground stations, rajectory effects, performance hisiory) and their inreractions had staristically significant impacts on the RSS position enror. The factor values used in the ANOVA erperiment were:

Navaids= (VOR, DME, LORAN, TACAN, GPS)

Number of Starionsm [One. Two, Three]

(Model 1)

Trajectory Typer (High-Performance, Commercia) Transporr, General Aviaion

Performance History = (Iniervals: I, II, III, IV)

Since each trajectory consises of four, 15-minure legs, the Performance History (or "time interval") factor refers to the RSS error obtained within each 15-minute time frame. Four single-station, six doublestation, and four triple-station hybrids were simulated using combinations of four different Stations (named Stations $A-D)(9,12]$.

The ANOVA technique was applied to the simulation results as follows: First, the mean value of the RSS position error and the variance using all simulation data were computed. The ANOVA model decomposes the variance into a sum of variances, each associated with a potentially contributing factor and factor interactions. For example, if a two-factor ANOVA experiment using navaid type and number of ground stations was performed, the total variation of the RSS position error in the complete set of simulation results would be:

$$
S S_{\text {TOTAL }}=S S_{\text {NAV }}+S S_{\text {STAT }}+S S_{\text {NAV.STAT INT }}
$$$$
+ \text { SSUNEXPLANED }
$$

Here, SSTotal is the total variation in the RSS position error based on all simulations, $S S_{\text {NAv }}$ is the variation in the RSS position error due to differences in navaid type, SS STAT is the variation in the RSS position error due to the number of ground stations, and SS NAV.STAT NT is the variation in the RSS position error due to interaction effects. SS UNEXPLANVED is the variation in the RSS position error that cannot be attributed to navaid type, the number of stations, or interactions berween these two factors.

Equation (1) was expanded for the four-factor navaid experiment defined by Model $1[9,12]$. Using the computed sums-of-squares, Scheffe multiple comparison tests were performed to identify the specific differences within the groups (e.g., RSS position error differences berween different navaid types).

\section{Extracting Rules Using Induction: The ID3 Algorithm}

The $\mathbb{D} 3$ Algorithm uses inductive inference to extract rules [14] from an example set. The problem space is described in terms of arcributes, where each atribute is characterized by a set of values that define the possible "states." For example, in Model 1, the navaid type and number of ground stations were shown to be atuributes affecting RSS position error. The $\mathrm{ID} 3$ algorithm defines the shape of the decision tree as it identifies the most imporant artribute at each decision node. The algorithm uses an Information-Theoretic Measure (ITM) that minimizes the number of tests (atribute nodes) necessary to define the decision tree. The $\mathbb{D} 3$ algorithm uses the ITM in a splitting strategy [14] to decide which attribute provides the most information from the example set. (See Refs. 9,12,14 for details of the ID3 algorithm.)

\section{Developing the ID3 Atribute Framework Using ANOVA} Results

The Model 1 covariance simulations were used to extract decision trees for the NSM Expert system. Eleven anributes were defined for the ID3 framework: Navaid type, trajectory leg, aircraft groundspeed, number of ground starions, minimum geodetic distance from station, maximum geoderic disunce from station, (Max - Min) distance on trajectory leg, maximum line-of-site angle from station, minimum lineof-sight angle from the station, direction of flight (approaching or receding) relative to station, and RSS position error class on previous rajectory leg. The $\mathrm{D} 3$ algorithro determined how these atributes were related to each other and to the final RSS position error.

The classification scheme chosen to represent the RSS position error endnode in the NSM decision trees is depicted in Table II. The velocity, distance, and line-of-sight angles were expressed in terms of ranges instead of individual values, so the expert system weights trends more heavily than specific examples. This makes the expen system more adaptable to new condiaions, because matches between the actual and knowledge-base cases can be obeained more frequently. 
Table II RSS Position Error Classification Scheme

[High]

\section{Accuracy}

[Medium]

[Low]

Error

Range

Error

N.

Code

Range,

Code

N. Mi.

Error

Range, Code

N. Mi.

$0.10-0.20$

$0.20-0.30$

$0.30-0.40$

$0.40-0.50$

$0.50-0.60$

$0.60-0.70$

$0.70-0.80$

$0.80-0.90$

$0.90-1.00$

c- 6

$c-7$
$c-8$

$c-8$

c-10

c-11

$c-12$

c-13

c-14

1.0-1.5

$1.5-2.0$

2.0-2.5

2.5-3.0

3.0-3.5

$3.5-4.0$

$4.0-4.5$

4.5-5.0

$>5.00$

c-15

c-16

$c-17$

c-18

c-19

$c-20$

$c-21$

c- 22

c-23

The example set was developed using the atribute framework described above. The RSS position errors for each simulation were classified on each trajectory leg using the scheme in Table II. The ID3 example base was then created from each single-, double-, and triplestation simulation.

\section{NSM Decision Trees}

The NSM example set was divided into 17 smaller example sets. The GPS and on-board navaid examples were grouped into one exper, whereas the ground-based navaid examples were divided according to navaid type and time (15-minute intervals). The $\mathbb{D} 3$ algorithm constructed decision trees for each of the 17 small expert systems to comprise the larger NSM Expert. The total number of examples used to develop the NSM Expert System was 932 , based on 260 Kalman Filter covariance simulations. An additional 37 simulations were performed to obtain an RSS error-estimate decision tree for navaid-type combinations. The NSM expert system prompts the user for a set of flight conditions commensurate with the attribute/value lists used in the example set. and the resulting RSS classification code is rerumed to the user from the decision tree.

Figure 3 shows decision trees for single-, double- and tripleTACAN station combinations on the second 15 -minure trajectory leg. Here, the majority of the testing nodes are trajectory parameters (distance, LOS angle, direction of flight with respect to the stations). The top or root node is the aircraft's direction of flight. This is expected because the distance and LOS angle attributes are dependent on directional motion. Distance, LOS angle, and groundspeed are results of the aircraft's motion; hence, they represent more specific problem parameters, and it is expected that these parameters appear deeper in the decision tree. Figure 3 also shows that distance, ground velocity, LOS angle, and hybrid performance history are significant factors in RSS error prediction.

The decision tree in Fig. 4 shows the expected position error range when lifferent navaid types are integrated into a hybrid system. The RSS position errors for these simulations were averaged over the entire flight time for the high-performance trajectory. The tree is organized in terms of the navigation method used: (1) Distance-Velocity $(p-V),(2)$ Bearing-Velocity $(\theta-V)$, (3) Distance-Bearing $(\rho-\theta)$, (4) DistanceDistance $(\rho-\rho)$, (5) Bearing-Bearing $(\theta-\theta)$, and (6) Velocity-Velocity (V-V). These results show that LORAN is a better distance-measuring navaid than DME and that Doppler Radar provides better navigation accuracy than the Air Data Sensor when $\rho$ - $V$ navigation is used. The $p-\theta$ results show that it is possible to obtain good perfomance when LORAN and VOR are used. The LORAN/DME hybrid gives better results than two DME stations but worse performance than two LORAN stations. By far the worst results are obtained using two VOR stations.

\section{Performance of NSM Expert on Test Trajectories}

Two high-performance trajectories were used in the performance evaluation of the NSM Exper. Multi-station hybrids were simulated on each test trajectory for the DME, VOR. TACAN, and LORAN systems. In total, 60 covariance simulations were performed for the

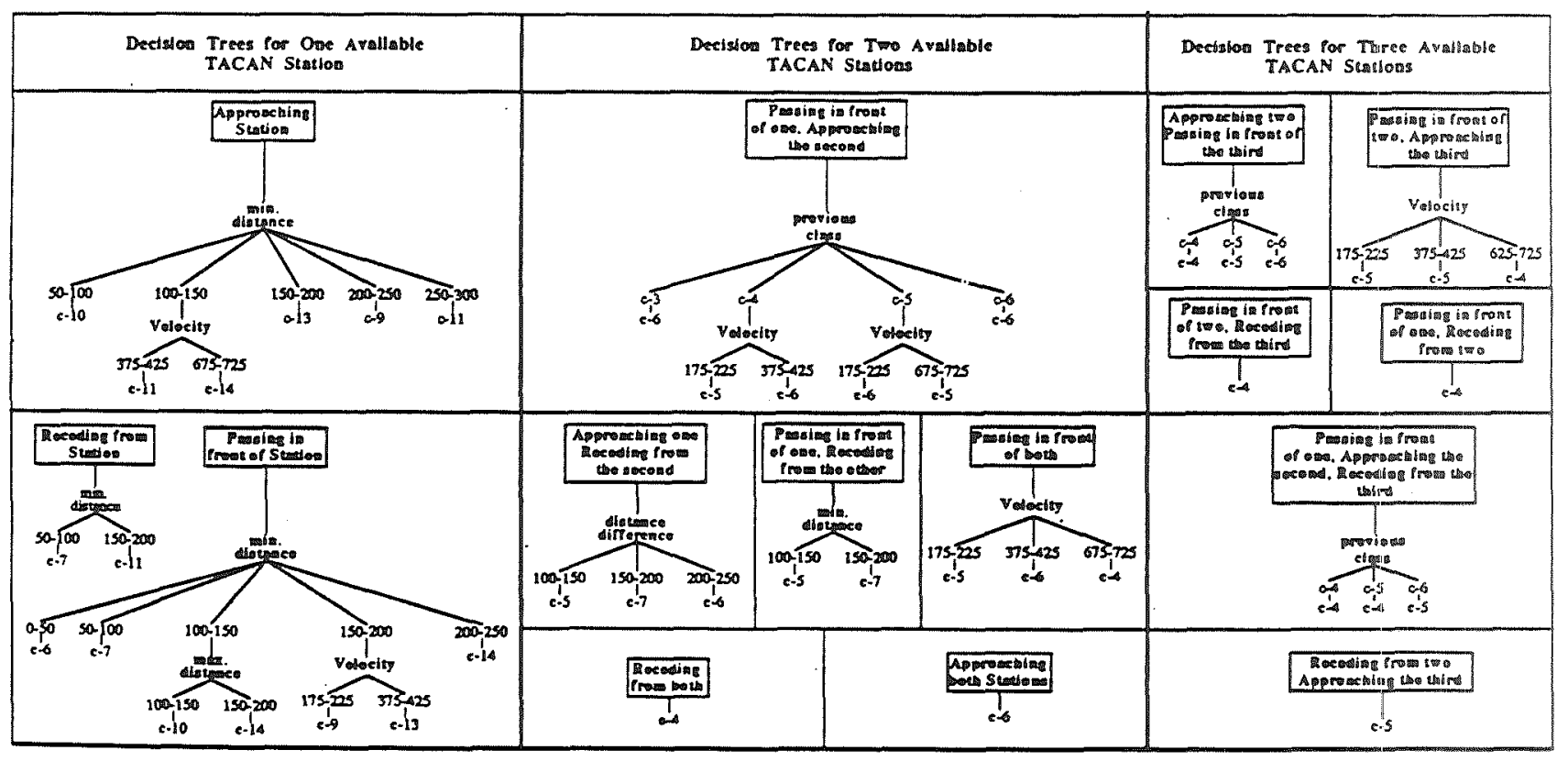

Figure 3. Decision Trees Predieting RSS Position Error Range for an INS Aided by TACAN During the Second 15 Minutes of Flight 


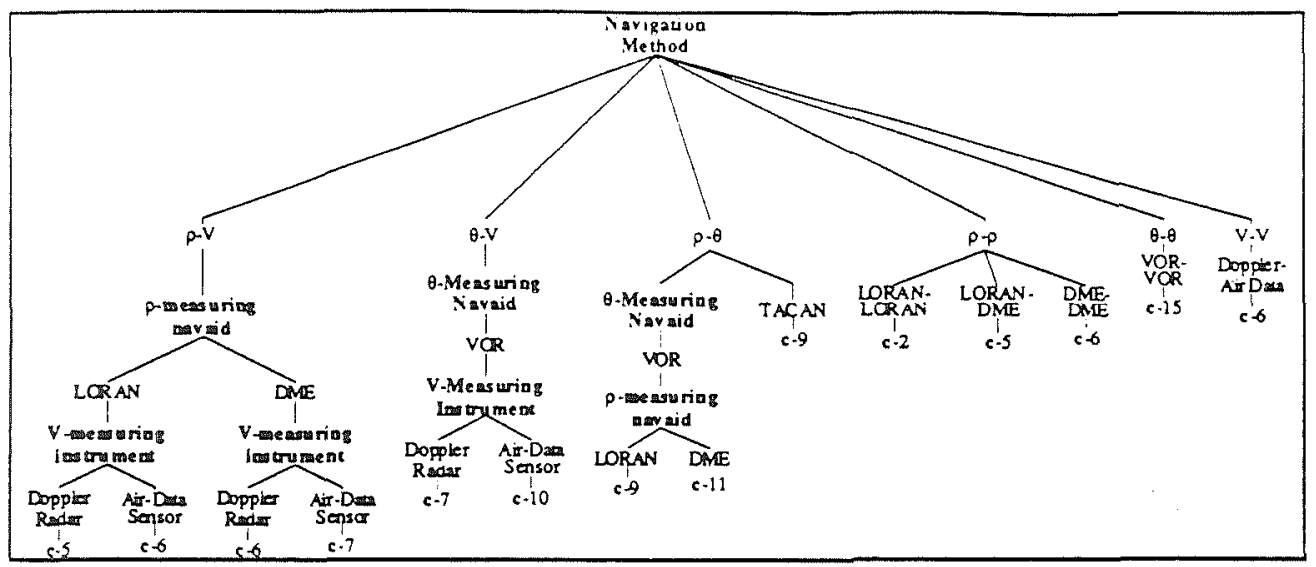

Figure 4. Decision Tree Predicting RSS Performance When Different Navaid Combinations are Used to Aid an INS

two test trajectories. The results for each simulation were classified on each trajectory leg according to the scheme in Table II. The total number of matches was counted on each trajectory leg for the seven navaid types studied. Figure 5 shows how well the NSM Expert predicts the RSS position ertor for each hybrid configuration. The predicrive performance metric for each navaid is defined as the percentage of number of matches obtained from the total number of combinations tested for that navaid.

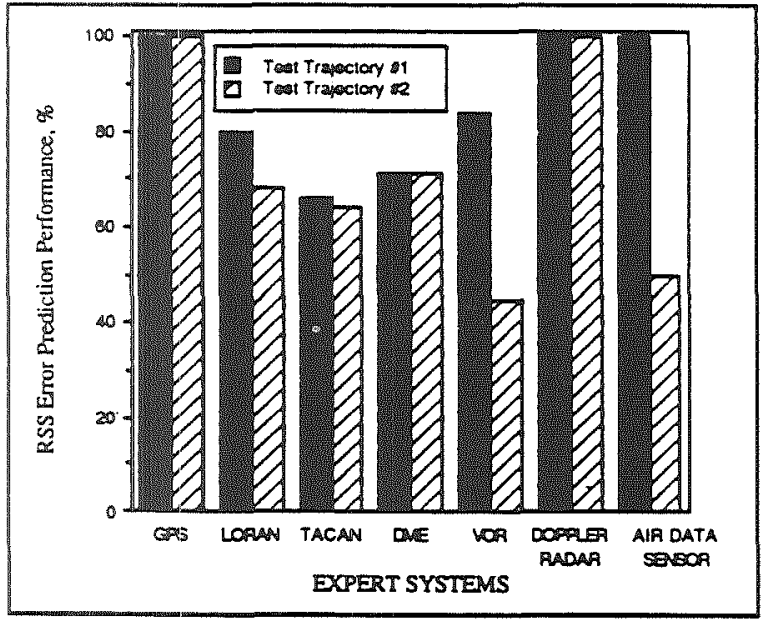

Figure 5. Performance of Navaid Experts on Test Trajectories

The NSM Expert performed very well on the two test trajectories. Figure 5 shows that the NSM Expert correctly predicts the RSS position error betrer than $70 \%$ of the time on test Trajectory \#1. The system required only the trajectory informacion and its knowledge of hybrid system performance to make these predictions. However, its predictive capabilizy on test Trajectory 2 is slightly worse for the LORAN hybrids (69\%), considerably worse for the VOR (45\%) and Air Daca sensor liybrids (53\%), and identical to the trajectory \#1 results for the remaining configurations. Hence, the results from Trajectory *2 suggest that additional investigation into trajectory effects on VOR's and Air Data Serssor's performance may be necessary.

Selecting Navigation Strategies Using the NSM Expert Sysiem

The NSM-Recommended Navaid strategies were compared with covariance-determined strategies for various navigation sensor suite scenarios $[9,12]$. For example, the NSM Expen was asked to find the best two-measurement hybrid strategies for the following navaid sensors:

Sensor Scenario \#1 = 12 GPS satellites, LORAN Station A. DME Station A, VOR Station C, Doppler Radar

The results obtained by mixing combinations of the Scenario \#1 sensors are shown in Fig. 6. The NSM Expert correctly identified the best, next-best, and third-best navigation strategies. Additional NSM Expert results are given elsewhere $[9,12]$

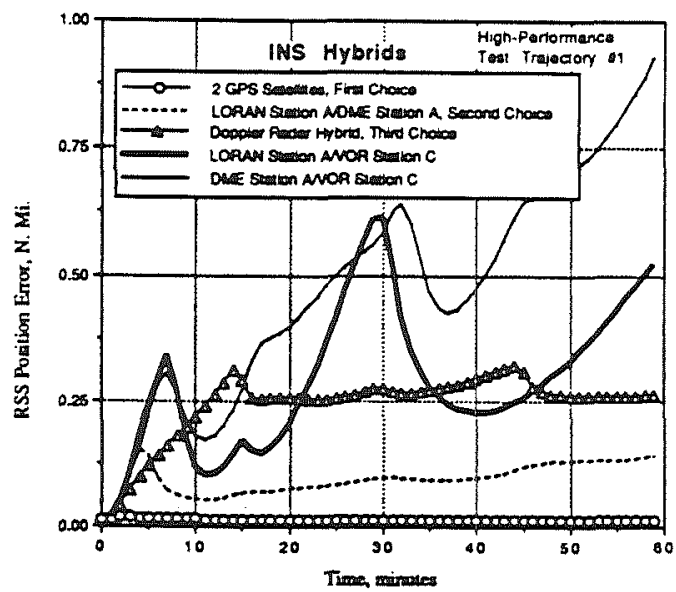

Fgure 6. NSM-Recommended Navaid Strategies

In addition to recommending navigation strategies from a given set of sensors, several heuristic sensor selection schernes were determined from the ANOVA results, the Scheffe comparisons, and the ID3 decision trees.

For single-type hybrids whose ground stations are at or near the same location, the ranking in order of best to worst performance was determined from ANOVA and the Scheffe tests as follows:

Ranking heuristic for = (GPS, LORAN or TACAN, DME, VOR) co-located navaids 


\section{REFERENCES}

The rarking scheme above applies to single-station hybrids (or doublesatellite GPS hybrids). The Scheffe comparisons show that a TACAN or LORAN selection depends on trajectory effects; this means that either a TACAN or LORAN hybrid could provide staristically lower RSS position errors depending on the aircraft flight path with respect to the stations being compared. The ranking heuristic is accurate with a $99 \%$ confidence level. If non-colocated starions are compared. trajectory effects must be considered for these single-station hybrids. The ANOVA and Scheffé comparison tests showed that double-station hybrids provide much smaller RSS position errors than single-station hybrids $[9,11,12]$. The performance difference between two and three stations is not as dramatic but is nonetheless statistically significant [12].

For multi-station hybrids of the same navaid type, the ANOVA and ID3 results provide the following ranking scheme from best to worst navigation performance as follows [9]:

Ranking heuristic for

mult-station hybrids $=14$ GPS, 3 GPS, 3 LORAN, 2 LORAN, and on-board sensor 3 TACAN, 2 TACAN, 3 DME, 2 DME, hybrids Doppler Radar, Air data sensor, 3 VOR 2 VOR \}

However, the decision trees highlight the importance of trajectory effects in RSS position error estimation.

\section{CONCLUSIONS}

This paper summarized two research efforts to develop expert systems using systematic methods: The design methodology for multiple cooperating rule-based systems, and the development of a navigation sensor management system. Nine modular rule-based systems, collectively called AUTOCREW, were designed to automate functions and decisions associated with a combat aircraft's subsystems. The nine AUTOCREW knowledge bases were designed individually and areas of cooperarion between the knowledge bases were identified. Each knowledge-base was designed using a graphical symbology that clearly illustrated the relationships between functional and decision-making tasks. Performance merrics were developed to evaluate the workload of each knowledge base and to assess the cooperation berween the rule-bases.

The NA VIGATOR's sensor management task was treated in detail. The performances of seven navigation systems aiding a mediumaccuracy Inertial Navigation System (INS) were investigated using Kalman Filter covariance analyses. A Navigation Sensor Management Expert System was formulated from covariance simulation data using the Analysis-of-Variance (ANOVA) method and the ID3 algorithm. ANOVA results show that statistically different position accuracies are obtained when different navaids are used, the number of navaids aiding the INS is varied, the aircraft's trajectory is varied, and the performance history is varied. The ID3 algorithm determines the NSM Experi's classification "rules" in the form of decision trees. The performances of these decision trees were assessed on two arbitrary trajectories, and the results demonstrate that the NSM Expert adapts to new situations and provides reasonable estimates of the expected hybrid performance. The NSM Expert demonstrates that carefullyplanned simulation experiments can be used to develop a fullyoperational expert system with a designer-specified performance effectiveness.

\section{ACKNOWLEDGMENTS}

This project was sponsored by the U.S. Army Research Office under Contract No. DAAG29-84-K-0048, and supported by NASA and the FAA under Grant No. NGL31-001-252. This work was partially supporied by the Natural Sciences and Research Council of Canada (NSERC) through the "1967" Scholarship. Lawrence Rosen's many helpful comments on this manuscript are also gratefully acknowledged.
[1] D.A. Handelman and R.F. Stengel, "A Theory for Fault-Tolerant Flight Conuol Combining Exper Sysiem and Analytical Redundancy Concepts", in Proceedings of the 1986 AIAA Guidance, Navigation, and Control Conference, 1986, pp. 375384.

[2] W.K. Ericksen, "The Blackboard Model: A Framework for Integrating Multiple Cooperaing Expert Systerns", in Proceedings of the 5th Computers in Aerospace Conference. 1985, pp. 33-40.

[3] F. Hayes-Roth, D. Waterman, and D. Lenal, Building Expert Systems, Addison-Wesley Publishing Company, Reading, MA., 1983.

[4] A.D. Pisarro and H.L. Jones, "An Exper System Approach to Adaptive Tactical Navigation", in Proceedings of The First Conference on Artificial Intelligence Applications, IEEE Computer Society, 1982, pp. 460-464.

[5] B.M. Anderson et al, "Intelligent Automation of Emergency Procedures in Advanced Fighter Aircraft", in Proceedings of The First Conference on Artificial Intelligence Applications, DEEE Computer Society, 1982, pp. 496-501.

[6] S.E. Cross, "Model-Based Reasoning in Expert Systems: An Application to Enroute Air Traffic Control", in Proceedings of the AIAAIIEEE 6th Digital Avionics Systems Conference, 1984, pp. 95-101.

[7] D.A. Handelman and R.F. Stengel, "An Architecture for RealTime Rule-Based Control", in Proceedings of the 1987 American Control Conference, 1987, pp. 1636-1642.

[8] E. Jablonski, Flying Fortress, Doubleday, Garden City, New York, 1965, pp. 324-339.

[9] B. L. Belkin, Cooperative Rule-Based Systems for Aircraft Navigation and Control, M.S.E. Thesi.s, Report 1856T, Department of Mechanical and Aerospace Engineering. Princeton University, June 1989.

[10] B.L. Belkin and R.F. Stengel, "Cooperative Rule-Based Systems For Aircraft Control", in Proceedings of the 26th IEEE Conference on Decision and Control, Los Angeies CA. December 1987, pp. $1934-1940$.

[11] B. L. Belkin and R. F. Stengel, "Quantitative Knowledge Acquisition for Expert Sysiems", Presented at the Space Operations, Applications, and Research Symposium, Albuquerque, NM, June 1990.

[12] B. L. Belkin and R. F. Stengel, "Knowledge Acquisition for Exper Systems Using Statistical Methods", Presented at the 51 st AGARD Symposium of the Guidance and Control Panel on Knowledge Based System Applications for Guidance and Control, Madrid, Spain, September 1990.

[13] G. E. P. Box, W. G. Hunter and J. S. Hunter, Statistics for Experiments: An Introduction to Design, Data Analysis and Model Building, John Wiley \& Sons, New York, 1978.

[14] J. R. Quinlan, "Discovering Rules by Induccion From Large Collections of Examples", in Expert Systems in the Micro Electronic Age, D. Michie, Editor, Edinburgh University Press, 1979, pp. 169-201.

[15] A. Gelb and A. Sutherland Jr., "Software Advances in Aided Inertial Navigation Systems", Navigation. The Instituse of Novigation, Washington DC, Vol. 17, No.4, Winter 1970-71, pp. 358-369.

[16] R. F. Stengel, Stochastic Optimal Control -- Theory and Application, J. Wiley \& Sons, New York, 1986. 